Poster \#9

\section{Franken Foods or Smart Foods? A New Language for Biotechnology?}

\author{
Glenn Mitchell, Wendy Russell \& Natalie Stoianoff
}

BELSA (Biotechnology - Ethical, Legal and Social Aspects) Research Group, University of Wollongong, Australia

In recent months, numerous articles about genetically modified food and the biotechnology industry have appeared in Australian newspapers. These have presented competing claims, not only about scientific aspects (e.g., health risk, environmental impact, risk of escape, etc.) but also about non-scientific aspects of the new gene technologies. A certain degree of rigour is demanded of scientific evidence, including, increasingly, information on health and environmental risk. In contrast, it seems that statements regarding the impacts of biotechnology on the economy, on food security, and on the needs of farmers and consumers are being made on the basis of judgement, opinion and untested assumption. Such a basis has contributed to heated, unproductive argument.

There is clearly a need for rigorous research in these areas, and while it is impossible to test for the long-term impacts of new technologies now, it is possible to make informed predictions based on accurate data and observations about the current situation. This information must be collected at micro as well as macro levels. For example, statistics about global population change and food production are often used in projections of the need for agricultural biotechnology for food security, yet without understanding the relationship between food production and food consumption, at national and community levels, these projections may be seriously flawed. They may also not address the root causes of hunger and poverty, many of which are related to patterns of land ownership and employment. It is arguably more important to understand how new technologies impact on these patterns.

Research must also investigate how political, legal and funding processes which regulate, promote and initiate biotechnology research respond to different imperatives, such as ecologically sustainable development and economic equity. It makes more sense for such imperatives to be operative from the earliest stage of research and development rather than just at the final stage of screening new technologies. Moreover, thorough and transparent policy processes, which allow public scrutiny and input, and which are seen to address safety, sustainability, utility and equity, are required to increase public confidence in these new technologies.
This paper will outline the current situation, and the history of policy development, in agbiotech in Australia. This will include a summary of work being done here in the area of social, legal and ethical aspects of agbiotech. The paper, as well as proposing research questions that need to be addressed in this area, will suggest that a new language, of both words and ideas, is needed to bridge discipline gaps and bring light to the debate on biotechnology.

Poster \#10

\section{Introgression and Persistence of an Insecticidal Transgene Can Be Monitored by GFP}

\author{
Matthew D. Halfhill \& C. Neal Stewart, Jr. \\ Department of Biology, UNC, Greensboro, NC 27402, USA
}

There are growing concerns about the introgression and persistence of transgenes into weedy wild relatives of crops. To investigate the risk of such an occurrence, we have transferred a fitness enhancing and insecticidal Bacillus thuringiensis (Bt crylAc) transgene to oilseed rape (canola) Brassica napus (AACC) and then by hybridization to the wild relative Brassica rapa (AA) Canola with $B t$ transgenes in various loci are introgressed into $B$. rapa from rates ranging from very high to zero, indicating that when transgenes are on the A genome, they can be easily moved into $B$. rapa, where they can persist. Transgenes located on the $\mathrm{C}$ genome are not able to be introgressed into B. rapa. Furthermore, transgenes are expressed at equitable levels in hybrids and $\mathrm{Bt}$ introgressed $B$. rapa as they are in the $B t$ canola parents. Therefore, we have constructed plasmids with linked $B t$ and green fluorescent protein (GFP) in plant transformation vectors to test the effectiveness of using GFP to monitor transgene flow and persistence in this system. Like $B t$, GFP expression is also at equivalent levels in hybrids and introgressed $B$. rapa as compared with the canola parent. These tools will enable upcoming large field experiments to be performed to better assess the ecology and consequences of transgene escape into wild relatives. 